\title{
Time and Space Efficient Spectral Clustering via Column Sampling
}

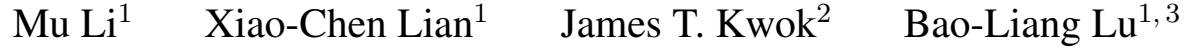 \\ ${ }^{1}$ Department of Computer Science and Engineering, Shanghai Jiao Tong University, Shanghai 200240, China \\ ${ }^{2}$ Department of Computer Science and Engineering, Hong Kong University of Science and Technology, Hong Kong \\ ${ }^{3}$ MOE-Microsoft Key Laboratory for Intelligent Computing and Intelligent Systems \\ Shanghai Jiao Tong University, Shanghai 200240, China \\ \{limu.cn, lianxiaochen\}@gmail.com, jameskecse.ust.hk, bllu@sjtu.edu.cn
}

\begin{abstract}
Spectral clustering is an elegant and powerful approach for clustering. However, the underlying eigendecomposition takes cubic time and quadratic space w.r.t. the data set size. These can be reduced by the Nyström method which samples only a subset of columns from the matrix. However, the manipulation and storage of these sampled columns can still be expensive when the data set is large. In this paper, we propose a time- and space-efficient spectral clustering algorithm which can scale to very large data sets. A general procedure to orthogonalize the approximated eigenvectors is also proposed. Extensive spectral clustering experiments on a number of data sets, ranging in size from a few thousands to several millions, demonstrate the accuracy and scalability of the proposed approach. We further apply it to the task of image segmentation. For images with more than 10 millions pixels, this algorithm can obtain the eigenvectors in 1 minute on a single machine.
\end{abstract}

\section{Introduction}

Spectral clustering has been widely used in diverse areas such as data mining [10, 5], signal processing [1] and computer vision [11, 12]. Given $n$ data points, it is based on the eigen-decomposition of an $n \times n$ matrix that is derived from the pairwise similarities of these points. For example, the normalized cut algorithm [12], which is the focus of this paper, computes the eigenvectors corresponding to the $k$ smallest eigenvalues of the normalized Laplacian matrix. Using the $k$ respective components of the data points in these eigenvectors, another clustering algorithm (e.g., the $k$-means) is then invoked to form the final clusters. However, the requirement of computing and storing the affinity matrix, and the $\mathcal{O}\left(n^{3}\right)$ complexity of decomposing the Laplacian matrix, severely restrict the scalability of spectral clustering to large data sets [12].

As only several eigenvectors are required in the proce- dure, a general approach to alleviate this problem is by using low-rank matrix approximations, among which the Nyström method $[4,17]$ is the most popular. It samples $m \ll n$ columns from the original $n \times n$ matrix, and then forms a low-rank approximation of the full matrix by using the correlations between the sampled columns and the remaining $n-m$ columns. As only a portion of the full matrix is computed and stored, the Nyström method can reduce the time and space complexities significantly. Fowlkes et al. successfully applied this to spectral clustering for image segmentation [6]. Besides this, the Nyström method has also been popularly used for tasks such as Gaussian processes [17] and manifold learning [15].

However, when the data set is huge, even the Nyström method can suffer from storage problems. Specifically, since the sampled columns have to be frequently used in the computational procedure, typically they have to be stored in the main memory. For data sets containing millions of samples, even storing only $1 \%$ of the columns (in double precision) takes about $80 \mathrm{~GB}$.

Another problem with the Nyström method is that the eigenvectors obtained are not orthogonal in general. As has been shown in [8], the lack of orthogonality can sometimes adversely affect the approximation quality. Thus, an additional orthogonalization step may have to be performed. However, in image segmentation, this is further complicated by that the affinity matrix used is typically sparsified and not positive semidefinite (psd). The corresponding orthogonalization procedure proposed in [6] then takes cubic time, making it impractical to implement in practice.

In this paper, we propose a time- and space-efficient spectral clustering algorithm based on the Nyström method. However, in contrast to the approach in [6], we directly compute a rank- $k$ approximation of $M=I-L$ (where $I$ is the identity matrix and $L$ is the normalized Laplacian matrix) using the sampled columns of the affinity matrix. With just one single pass on the sampled columns, both the approximated eigenvectors of $M$ and the affinity degrees can 
be computed simultaneously. Thus, these sampled columns no longer have to be stored, alleviating the storage problem when $n$ is very large. We further propose a general algorithm to orthogonalize the approximated eigenvectors, irrespective of whether the underlying matrix is psd or not.

Our contributions are twofold. First, we reduce the time and space complexities of the existing Nyström-based spectral clustering algorithm [6]. Theoretical analysis shows that the proposed algorithm can exactly recover the degrees of the sampled points. Experimentally, the algorithm can scale to much larger data sets (with millions of samples) than the existing algorithms. Second, we apply the proposed algorithm to the segmentation of images with very sparse affinity matrices. We demonstrate its scalability on images with more than 10 million pixels. To our best knowledge, this is the first attempt to scale spectral clustering to such a large image segmentation task.

The rest of this paper is organized as follows. Section 2 briefly reviews spectral clustering and the Nyström based approach. Section 3 then describes the proposed algorithm. Experimental results are presented in Section 4, and Section 5 gives some concluding remarks.

Notations. For a symmetric matrix $A \in \mathbb{R}^{n \times n}$, we denote by $A_{k}=U \Lambda U$ its rank- $k$ approximation, where $\Lambda$ contains its $k$ largest eigenvalues and $U$ contains its $k$ leading eigenvectors. Moreover, $\operatorname{diag}(v)$ is the matrix with the elements of $v$ on the main diagonal.

\section{Related Works}

\subsection{Spectral Clustering}

Spectral clustering is based on analyzing the affinity matrix $A=\left[a_{i j}\right] \in \mathbb{R}^{n \times n}$ defined on a set of $n$ data points $X=\left\{x_{1}, \ldots, x_{n}\right\}$, where $a_{i j} \geq 0$ is the similarity / affinity between $x_{i}$ and $x_{j}$. In general, there are several ways to group $X$ into clusters [16]. In this paper, we focus on the popular method of normalized cut [12]. It first computes the normalized Laplacian $L=I-D^{-\frac{1}{2}} A D^{-\frac{1}{2}}$ of $A$, where $D=\operatorname{diag}(A 1)$ is the degree matrix and 1 is the vector of all ones. Next, the $k$ trailing eigenvectors of $L$ are obtained, where $k$ is usually not less than the number of clusters. Finally, $k$-means clustering is performed on these eigenvectors to obtain the clusters.

\subsection{Nyström Method}

The Nyström method [4, 17] has been commonly used to construct low-rank matrix approximations. Given a symmetric matrix $A \in \mathbb{R}^{n \times n}$, it first samples $m \ll n$ columns from $A$. Without loss of generality, we can assume these to be the first $m$ columns of $A$, and thus $A$ can be rewritten as

$$
A=\left[\begin{array}{ll}
A_{11} & A_{21}^{T} \\
A_{21} & A_{22}
\end{array}\right] \text { and } A_{: 1}=\left[\begin{array}{l}
A_{11} \\
A_{21}
\end{array}\right]
$$

where $A_{: 1} \in \mathbb{R}^{n \times m}$ is the submatrix containing the selected columns, and $A_{11} \in \mathbb{R}^{m \times m}$ is the submatrix containing the intersection of the selected rows and columns. The Nyström approximation of $A$ is then given by

$$
\hat{A}_{\text {nys }}=A_{: 1} A_{11}^{-1} A_{: 1}^{T} \text {. }
$$

\subsection{Using Nyström in Spectral Clustering}

The normalized cut requires the computation of the $k$ trailing eigenvectors of the normalized Laplacian $L=I-$ $D^{-\frac{1}{2}} A D^{-\frac{1}{2}}$, which are the same as the $k$ leading eigenvectors of the $n \times n$ matrix

$$
M=D^{-\frac{1}{2}} A D^{-\frac{1}{2}} .
$$

The direct eigen-decomposition of $M$ takes $\mathcal{O}\left(n^{3}\right)$ time, and can be expensive when $n$ is large. In [6], this problem is alleviated by using the Nyström method to approximate the eigenvectors as follows. First, to avoid using the whole $A$, the degree matrix $D$ is approximated as

$$
\hat{D}=\operatorname{diag}\left(\hat{A}_{\text {nys }} 1\right)=\operatorname{diag}\left(A_{: 1} A_{11}^{-1} A_{: 1}^{T} 1\right),
$$

by using the Nyström approximation of $A$ in (2). Let $D_{: 1}=$ $\operatorname{diag}\left(A_{: 1}^{T} 1\right)$. The submatrices in $M$ corresponding to $A_{11}$ and $A_{: 1}$ are

$$
M_{11}=D_{: 1}^{-\frac{1}{2}} A_{11} D_{: 1}^{-\frac{1}{2}}, \quad M_{: 1}=D^{-\frac{1}{2}} A_{: 1} D_{: 1}^{-\frac{1}{2}} .
$$

Using $\hat{D}, M_{: 1}$ can then be approximated as

$$
\hat{M}_{: 1} \simeq \hat{D}^{-\frac{1}{2}} A_{: 1} \hat{D}_{: 1}^{-\frac{1}{2}}=\left[M_{11} \hat{M}_{21}^{T}\right]^{T}
$$

and $M$ as

$$
\hat{M}=\hat{M}_{: 1} M_{11}^{-1} \hat{M}_{: 1}^{T},
$$

where $\hat{M}_{: 1}$ now contains the approximate eigenvectors of $M$.

In general, $\hat{M}_{: 1}$ is not orthogonal. To obtain better clustering results, Fowlkes et al. [6] proposed the following orthogonalization procedure when $A$ is psd. First, construct

$$
S=M_{11}+M_{11}^{-\frac{1}{2}} \hat{M}_{21}^{T} \hat{M}_{21} M_{11}^{-\frac{1}{2}} .
$$

Then, perform singular value decomposition (SVD) on $S$ to obtain $U \Lambda T^{T}$. The orthogonal decomposition of $\hat{M}$ can be obtained as $\hat{M}=V \Lambda V^{T}$ and $V^{T} V=I$, where

$$
V=\hat{M}_{: 1} M_{11}^{-\frac{1}{2}} U \Lambda^{-\frac{1}{2}} .
$$

The whole algorithm is summarized in Algorithm 1. When $A$ is not psd, a more complicated two-step orthogonalization step has to be used [6].

The time complexity of Algorithm 1 is $\mathcal{O}\left(n m^{2}+m^{3}\right)$. As for space, since $A_{: 1}$ and $\hat{M}_{: 1}$ need to be accessed more than once (e.g., in (4), (5), (6), (7), (8)), typically they have 


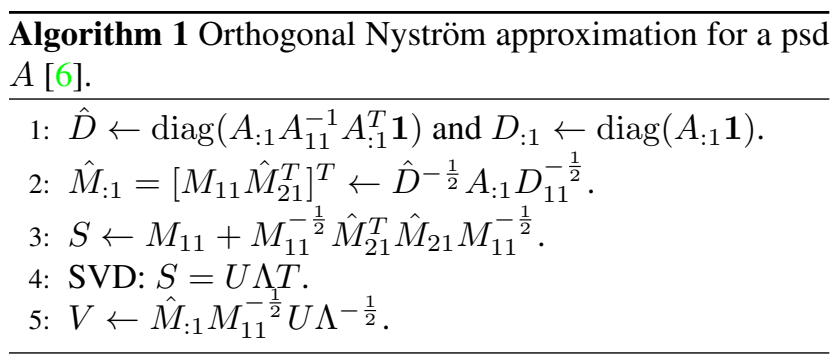

to be stored in main memory so as to avoid duplicate computations. However, this leads to $\mathcal{O}(\mathrm{nm})$ space complexity. In large-scale applications, even the sampling of a small subset of columns can result in the matrices $A_{1}$ and $\hat{M}_{: 1}$ being too large to fit into main memory. Moreover, when $A$ is not psd, the two-step orthogonalization procedure, which has a time complexity $\mathcal{O}\left(n^{3}\right)$, is often impractical [6].

\section{Proposed Approach}

Recall that spectral clustering only needs the $k$ trailing eigenvectors of the normalized Laplacian $L$, or, equivalently, the $k$ leading eigenvectors of $M=I-L$. Therefore, our goal is to find a rank- $k$ approximation $M_{k}$ of $M$. Let the eigen-decomposition of $M_{k}$ be $U \Lambda U^{T}$, where $\Lambda$ contains the $k$ largest eigenvalues of $M$, and $U$ contains the corresponding eigenvectors. As $M_{k}=M M_{k}^{-1} M$, so on using (3), we have

$$
\begin{aligned}
M_{k} & =D^{-1 / 2} A D^{-\frac{1}{2}} M_{k}^{-1} D^{-\frac{1}{2}} A^{T} D^{-1 / 2} \\
& =D^{-1 / 2} A S_{A} A^{T} D^{-1 / 2}
\end{aligned}
$$

where

$$
S_{A}=D^{-\frac{1}{2}} M_{k}^{-1} D^{-\frac{1}{2}}
$$

is a rank- $k$ matrix. Note that although this $M_{k}$ is the best rank- $k$ approximation of $M$, (9) requires the use of the whole affinity matrix $A$. This can be too expensive for large data sets.

\subsection{A Good Approximate Degree Matrix}

Note that $A S_{A} A^{T}$ in (9) plays the role of the affinity matrix $A$ in (3). Since the handling of $A$ is expensive for large data sets, we consider in the following an approximation which only involves the $m$ sampled columns $A_{: 1}$ as in the Nyström method. As will be seen, this resultant approximation is in the form of $A S_{A} A^{T}$, and captures most of the information available in $A$. A comparison with some other possible approximations will be discussed in Section 3.4.

Recall that the definition of $S_{A}$ in (10) relies on $M_{k}$, which in turn is the rank- $k$ approximation of $M$ in (9). Hence, we first define an analogous matrix $M_{*} \in \mathbb{R}^{m \times m}$ which is based on the submatrix $A_{11}$ :

$$
M_{*}=D_{*}^{-\frac{1}{2}} A_{11} D_{*}^{-\frac{1}{2}},
$$

where $D_{*}=\operatorname{diag}\left(A_{11} 1\right)$. Analogous to the definition of $S_{A}$ in (10), we then define the following matrix $S_{*} \in$ $\mathbb{R}^{m \times m}$ based on the rank- $k$ approximation $M_{*, k}$ of $M_{*}$ :

$$
S_{*}=D_{*}^{-\frac{1}{2}} M_{*, k}^{-1} D_{*}^{-\frac{1}{2}} .
$$

Obviously, $S_{*}=S_{A}$ when $m=n$. Finally, define, in a manner analogous to (9),

$$
\hat{A}=A_{: 1} S_{*} A_{: 1}^{T} .
$$

The following proposition shows that $\hat{A}$ can exactly recover the degrees of the $m$ sampled columns. The proof is in Appendix A.

Proposition 1. Assume that graph $G$ has no more than $k$ connected components. Then $A_{: 1} 1=\hat{A}_{: 1} 1$.

In other words, this approximation $\hat{A}$ contains all the degree information about the selected columns that is in $A$. The assumption of Proposition 1 is reasonable since $k$ is typically chosen not less than the number of connected components; otherwise we obtain $k$ eigenvectors according to the 0 eigenvalue of $L$ and are not unique [16]. Moreover, $\hat{A}$ also has a close relationship with the Nyström approximation $\hat{A}_{\text {nys }}$ of $A$ in (2). Specifically, when $k=m$, we have $\hat{A}=A_{: 1} D_{*}^{-\frac{1}{2}} M_{*}^{-1} D_{*}^{-\frac{1}{2}} A_{: 1}^{T}=A_{: 1} A_{11}^{-1} A_{: 1}^{T}=\hat{A}_{\text {nys }}$ on using (11). Thus, $\hat{A}$ is similar to $\hat{A}_{\text {nys }}$, but with a further rank- $k$ approximation on $M_{*}$.

\subsection{Rank- $k$ Approximation of $M$}

Let the eigen-decomposition of $M_{*, k}$ be $V \Lambda V^{T}$, where $V \in \mathbb{R}^{m \times k}$ and $\Lambda \in \mathbb{R}^{k \times k}$. On combining this with (12) and (13), we obtain $\hat{A}=Q \Lambda Q^{T}$, where

$$
Q=A_{: 1} D_{*}^{-\frac{1}{2}} V \Lambda^{-1}
$$

Since this $\hat{A}$ contains all the degree information about the selected columns, we can approximate the degree matrix as

$$
\hat{D}=\operatorname{diag}(\hat{A} 1)=\operatorname{diag}\left(Q \Lambda Q^{T} 1\right) .
$$

Subsequently, a rank- $k$ approximation of $M$ can be obtained as

$$
\hat{M}_{k}=\hat{D}^{-\frac{1}{2}} \hat{A} \hat{D}^{-\frac{1}{2}}=\hat{D}^{-\frac{1}{2}} Q \Lambda Q^{T} \hat{D}^{-\frac{1}{2}}=U \Lambda U^{T}
$$

where $U=\hat{D}^{-\frac{1}{2}} Q$ contains its eigenvectors. Note that although the derivation of $U$ above involves $\hat{A}$, it can be directly obtained from $Q$ without first forming $\hat{A}$.

When the data set is large, storage of the affinity matrix $A$, or even its submatrix $A_{: 1}$, can become infeasible. Fortunately, note that, here, $A_{: 1}$ is only needed once in the computation of $Q$ in (14). Thus, instead of storing the whole $A_{: 1}$ in memory, we can obtain $Q$ row by row. For its $i$ th 
row, we first compute the $i$ th row of $A_{: 1}$ (which contains the affinities between pattern $i$ and the sampled patterns in $A_{: 1}$ ), and then multiply it by $D_{*}^{-\frac{1}{2}} V \Lambda^{-1}$. Only the $n \times k$ matrix $Q$ needs to be stored. The whole algorithm is shown in Algorithm 2.

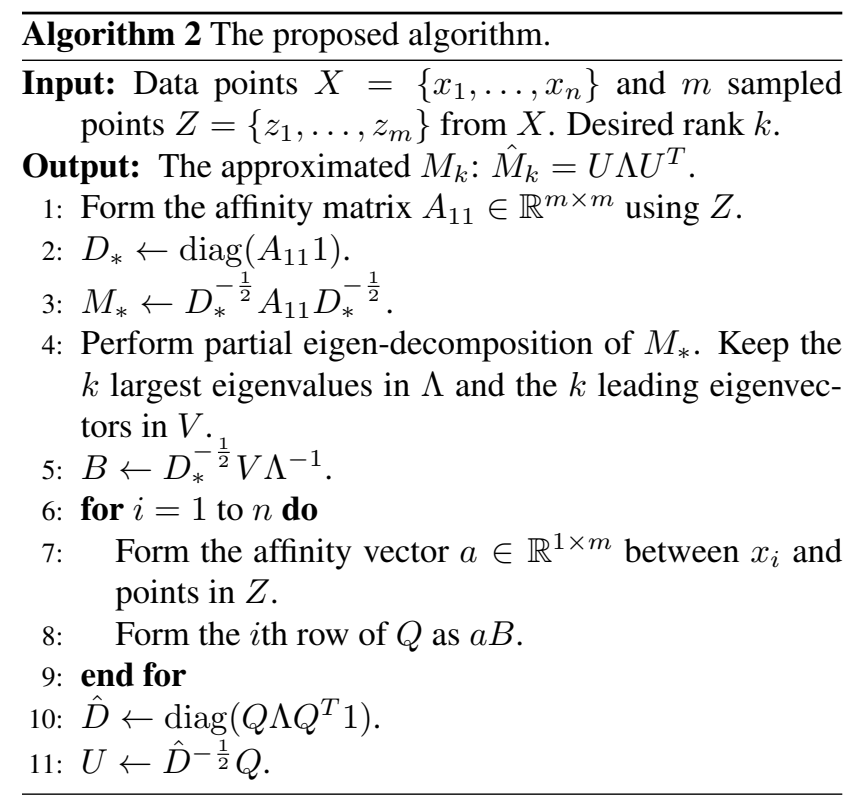

\subsection{Time and Space Complexities}

Since only partial eigen-decomposition is used in Algorithm 2, it takes $\mathcal{O}(n m k)$ time to compute $k$ approximate eigenvectors. In contrast, as the procedure in [6] needs to compute $A_{11}^{-1}$, it will take $\mathcal{O}\left(n m k+m^{3}\right)$ time instead.

As for the space complexity, when the matrix $A_{: 1}$ is small, it can be stored in main memory and so takes $\mathcal{O}(\mathrm{nm})$ space. However, when $n$ is large, $A_{: 1}$ may not be able to fit into memory. As discussed in the previous section, since the proposed algorithm only needs to access $A_{: 1}$ once, we can compute it row by row. The space complexity is then reduced to $\mathcal{O}(n k)$. On the other hand, Algorithm 1 needs to use $A_{: 1}$ more than once. To avoid computing $A_{: 1}$ repeatedly, we will have to store it in memory, leading to $\mathcal{O}(\mathrm{nm})$ space. A comparison of the time and space complexities between [6] and the proposed algorithm is shown in Table 1.

\begin{tabular}{|c|c|c|c|c|}
\hline \multirow{2}{*}{} & \multicolumn{2}{|c|}{ un-orthogonalized } & \multicolumn{2}{c|}{ orthogonalized } \\
\cline { 2 - 5 } & Fowlkes et al. [6] & ours & Fowlkes et al. [6] & ours \\
\hline time & $\mathcal{O}\left(n m k+m^{3}\right)$ & $\mathcal{O}(n m k)$ & $\mathcal{O}\left(n m^{2}+m^{3}\right)$ & $\mathcal{O}(n m k)$ \\
\hline space & $\mathcal{O}(n m)$ & $\mathcal{O}(n k)$ & $\mathcal{O}(n m)$ & $\mathcal{O}(n k)$ \\
\hline
\end{tabular}

Table 1 . Time and space complexities for computing $k$ approximate eigenvectors.

\subsection{Comparison with Other Approximations}

Other ways to obtain a rank- $k$ approximation of $M$ are possible. A straightforward approximation can be obtained by first forming the rank- $k$ Nyström approximation $\hat{A}^{(1)}=A_{: 1} A_{11, k}^{-1} A_{: 1}^{T}$ of $A$. Then, using (3), form a rank$k$ approximation of $M$ as $\hat{M}_{k}^{(1)}=\left(\hat{D}^{(1)}\right)^{\frac{1}{2}} \hat{A}^{(1)}\left(\hat{D}^{(1)}\right)^{\frac{1}{2}}$, where $\hat{D}^{(1)}=\operatorname{diag}\left(\hat{A}^{(1)} 1\right)$ is the approximate degree matrix. However, note that even when we sample all the $n$ columns from $A$ to form $A_{: 1}, \hat{M}_{k}^{(1)} \neq M_{k}$ in general and hence this cannot be expected to be a good approximation.

Another approximation, motivated from (10), is to use

$$
S^{(2)}=D_{: 1}^{-\frac{1}{2}} M_{11, k}^{-1} D_{: 1}^{-\frac{1}{2}}
$$

instead of $S_{*}$ in (12). Here, $D_{: 1}=\operatorname{diag}\left(A_{: 1} 1\right)$. It can be shown that the corresponding approximation of $M$ becomes $\hat{M}_{k}^{(2)}=\hat{M}_{: 1} M_{11, k}^{-1} M_{: 1}^{T}$, which is similar to the Nyström approximation of $M$ in (6) except for using a rank- $k$ approximation on $M_{11}$. However, with this approximation, we will then need to access $A_{: 1}$ twice, once to form $D_{: 1}$ and another to calculate $Q$ in (14). Subsequently, to avoid computing $A_{: 1}$ twice, we will need $A_{: 1}$ to be stored, which takes $\mathcal{O}(n m)$ space and can be infeasible when $n$ is large.

Moreover, unlike $S_{*}$ proposed in Section 3.1, these two approximations may not recover the degrees of $A_{: 1}$ (as in Proposition 1). Empirical results in Section 4.2 confirm that these two alternatives are less accurate.

\subsection{Orthogonalizing the Approximate Eigenvectors}

Note that the approximate eigenvectors $U$ obtained in (15) may not be orthogonal. In this section, we propose an inexpensive orthogonalization procedure which can transform any decomposition of the form $T=U \Lambda U^{T}$ to $T=$ $\tilde{U} \tilde{\Lambda} \tilde{U}^{T}$, such that $\tilde{U}$ is orthogonal (i.e., $\tilde{U}^{T} \tilde{U}=I$ ). Our procedure is more general than Algorithm 1 in that it does not require $T$ or $\Lambda$ to be psd.

The orthogonalization procedure is shown in Algorithm 3. Note that $P=U^{T} U$ is always psd and so it is safe to use $\Sigma^{\frac{1}{2}}$ and $\Sigma^{-\frac{1}{2}}$. The following proposition shows the correctness of Algorithm 3.

Proposition 2. In Algorithm 3, $U \Lambda U^{T}=\tilde{U} \tilde{\Lambda} \tilde{U}^{T}$ and $\tilde{V}^{T} \tilde{V}=I$.

It can be easily seen that the time complexity of Algorithm 3 is $\mathcal{O}\left(n k^{2}\right)$ and its space complexity is $\mathcal{O}(n k)$. Thus, unlike the orthogonalization procedure in [6], this does not increase the time complexity of the proposed algorithm (Table 1). 


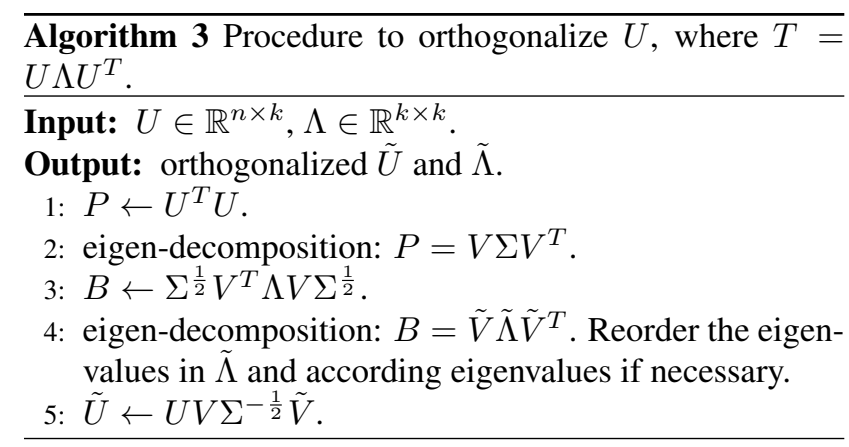

\section{Experiments}

\subsection{Spectral Clustering}

In this section, we perform spectral clustering experiments on a number of data sets with various sizes (Table 2). The USPS, MNIST and MNIST8M ${ }^{1}$ data sets contain handwritten digit images, with the raw pixels as features. We select the digits $0-4$ for MNIST and MNIST8M. The RCV1 ${ }^{2}$ is an extensively used text data set. It contains categorized news from Reuters, and each news article is represented by a sparse log-transformed TF-IDF feature vector [9]. After removing news categories with fewer than 1,000 articles, we obtain 55 categories that are used in the experiment. The ImageNet ${ }^{3}$ is an image database containing images organized according to the WordNet hierarchy. Each image is represented as a bag of words based on the SIFT features. We select the 100 largest categories from the training set. Each of these contains an average of 2,000 images.

For the ImageNet data set, we use the histogram intersection kernel [14], which has been commonly used on SIFT features, to construct the affinity matrix. For the other data sets, we use the Gaussian kernel $\exp \left(-\mu\|x-y\|^{2}\right)$, where $\mu$ is set to the inverse of the average distance among all data pairs (i.e. $\mu^{-1}=\frac{1}{n^{2}} \sum_{i j}\left\|x_{i}-x_{j}\right\|^{2}$ ).

\begin{tabular}{|c|c|c|c|c|}
\hline size & data & \#samples & dim & \#clusters \\
\hline \multirow{2}{*}{ small } & USPS & 9,298 & 256 & 10 \\
& MNIST & 35,735 & 784 & 5 \\
\hline \multirow{2}{*}{ medium } & RCV1 & 160,633 & 47,236 & 55 \\
& image-net & 191,050 & 1,000 & 100 \\
\hline large & MNIST8M & $4,130,460$ & 784 & 5 \\
\hline
\end{tabular}

Table 2. Data sets used in the spectral clustering experiments.

\footnotetext{
$1_{\text {http://www.csie.ntu.edu.tw/ cjlin/libsvmtools/ }}$ datasets/

${ }^{2}$ http://alumni.cs.ucsb.edu/ wychen/sc

$3_{\text {http: / / ww . image-net.org/challenges/LSVRC / }}$ 2010/download-public
}

\subsubsection{Setup}

The following spectral clustering methods will be compared:

1. Normalized cut algorithm [12] (denoted ncut).

2. $k$-means-based approximate spectral clustering [18] (denoted kasp).

3. Standard Nyström-based spectral clustering [6] (denoted psc).

4. The proposed algorithm (denoted ours).

The kasp is implemented ${ }^{4}$ in R, while the others are in Matlab. The implementation for psc is from the highly optimized code used in $[2]^{5}$. The kasp and psc implementations can directly output the clustering results. For ncut and ours, after dropping the leading eigenvector of $M$, we perform $k$-means clustering using the remaining $c$ leading eigenvectors, and with each data row normalized to unit norm. As the $c+1$ leading eigenvectors are required, we set $k=c+1$ for ours.

Recall that both psc and ours are based on column sampling. The number of sampled columns is fixed at $m=$ 1,000 , and these columns are selected by uniform sampling without replacement.

The following measures are used for performance evaluation: (1) accuracy; (2) normalized mutual information (NMI) [13], whose value is always between zero and one, and the higher the better; and (3) time. Moreover, we follow the common practice of permuting the obtained clusters to best match the ground truth. To reduce statistical variability, results for all the methods are averaged over 10 repetitions. All experiments, including the image segmentation in Sec. 4.2, are performed on a machine with Intel Xeon X5540 CPU and 16GB memory.

\subsubsection{Results on Small and Medium-Sized Data Sets}

Table 3 shows the results on the small data sets. The variances of the time measurements are very small and so are not reported. Moreover, only the orthogonalized versions of psc and ours are shown. Those for the un-orthogonalized versions are similar. As can be seen, the three methods sc, psc and ours, yield very similar accuracies and NMI values, while kasp is much inferior. This may be due to that a fixed kernel parameter $(\mu)$ has been used in our experiment, while the kasp experiments in [18] search $\mu$ over a range of possible values. As for the time, ours is faster than the others. Recall that the kasp implementation is in $\mathrm{R}$ while the others are in Matlab. Hence, the time comparison for kasp is for reference only.

\footnotetext{
${ }^{4}$ http: //www. stat. berkeley.edu/ dhyan/fasp.html.

5 http://alumni.cs.ucsb.edu/ wychen/sc.html
} 


\begin{tabular}{|c|c|c|c|r|r|}
\hline \multirow{2}{*}{ data } & \multirow{2}{*}{ method } & \multirow{2}{*}{ accuracy $(\%)$} & \multirow{2}{*}{ NMI } & \multicolumn{2}{|c|}{ time $(\mathrm{sec})$} \\
\cline { 5 - 6 } & & & & eig & total \\
\hline \hline \multirow{4}{*}{ USPS } & ncut & $\mathbf{7 0 . 3 2} \pm \mathbf{0 . 1 3}$ & $\mathbf{0 . 6 5 8} \pm \mathbf{0 . 0 0 1}$ & 4.2 & 8.5 \\
& kasp & $45.63 \pm 3.83$ & $0.395 \pm 0.013$ & - & 8 min \\
& psc & $68.14 \pm 0.11$ & $0.634 \pm 0.001$ & 28.9 & 30.0 \\
& ours & $70.00 \pm 0.61$ & $0.654 \pm 0.009$ & $\mathbf{2 . 9}$ & $\mathbf{3 . 5}$ \\
\hline \multirow{4}{*}{ MNIST } & ncut & $\mathbf{8 1 . 3 7} \pm \mathbf{0 . 1 5}$ & $\mathbf{0 . 6 3 6} \pm \mathbf{0 . 0 0 1}$ & 51.4 & 289.4 \\
& kasp & $59.08 \pm 6.25$ & $0.439 \pm 0.045$ & - & $40 \mathrm{~min}$ \\
& psc & $80.62 \pm 0.18$ & $0.619 \pm 0.001$ & 39.1 & 46.2 \\
& ours & $80.88 \pm 1.42$ & $0.624 \pm 0.015$ & $\mathbf{2 . 5}$ & $\mathbf{6 . 7}$ \\
\hline
\end{tabular}

Table 3. Clustering results on the small data sets. Here, "eig" is the time spent on computing the eigenvectors of the normalized Laplacian.

On the medium-sized data sets, ncut fails to run because of insufficient memory. As for kasp, its implementation does not allow use of the sparse data format and histogram intersection kernel. Hence, it is also dropped from the comparison. Results comparing the orthogonalized and un-orthogonalized versions of psc and ours are shown in Table 4. As can be seen, the use of orthogonalized eigenvectors improves the clustering results. However, this nearly doubles the time for psc, while that for ours is only increased by a very small fraction.

\subsubsection{Results on Large Data Sets}

The proposed method is particularly useful on large data sets. Using the MNIST8M data set, Figure 1 shows how the time for computing the orthogonalized approximate eigenvectors scales with number of samples $n$. As can be seen, ours grows much slower than psc. Moreover, psc suffers from insufficient memory when $n>10^{6}$. These agree with the complexity results discussed in Table 1. Detailed clustering results on a subset of $10^{6}$ samples and on the full set are shown in Table 5.

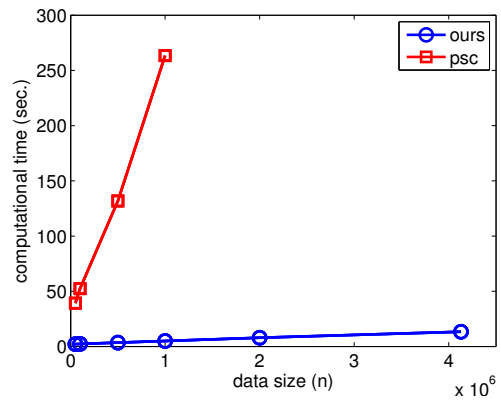

Figure 1. Time for computing the approximate eigenvectors versus data set size.

\subsection{Image Segmentation}

In this section, we evaluate our method on image segmentation. The approach in [7] is adopted to compute the affinity matrix. It uses a multi-layer graph $G^{*}=$ $\left(V^{*}, E^{*}, W^{*}\right)$, where the nodes $V^{*}=V \cup\left\{V^{(l)}\right\}_{l=1}^{L}$ are a set of pixels $V$ and the sets of regions $\left\{V^{(l)}\right\}_{l=1}^{L}, E^{*}$ is the edge set and $W^{*}$ is the weights on $E^{*}$. The node subset $V^{(l)}$ at the $l$ th layer corresponds to the over-segmentation result generated by the mean shift algorithm [3]. Different layers has different mean shift parameters. An undirected edge $e_{i j} \in E^{*}$ exists if (1) two pixels $i, j$ are within a neighborhood of radius $\alpha$; or (2) two regions $i, j \in V^{(l)}$ share a common boundary at the same layer; or (3) a pixel $i \in V$ is included in its corresponding region $j \in V^{(l)}$. This edge $e_{i j}$ then has the following weight $w_{i j} \in W^{*}$ :

$$
w_{i j}= \begin{cases}\exp \left(-\theta_{g}\left\|g_{i}-g_{j}\right\|\right), & i, j \in V \&\left\|x_{i}-x_{j}\right\| \leq \alpha \\ \exp \left(-\theta_{g}\left\|\bar{g}_{i}-\bar{g}_{j}\right\|\right), & i, j \in V^{(l)} \\ \gamma, & i \in V, j \in V^{(l)}\end{cases}
$$

where $x_{i}$, and $g_{i}$ are the position and color of pixel $i$, respectively, $\bar{g}_{i}$ is the mean color of inner pixels of region $i$, and $\theta_{g}$ is a constant that controls the strength of the weight. Note that in (17), the boundary cue is implicitly incorporated in the third case and $\gamma$ controls the trade-off between color and boundary cues. To perform segmentation, we apply the proposed method on $W^{*}$. We use grid sampling with step size $\alpha$ on images. Region nodes are not involved in the sampling step so as to avoid zero rows in the resulting matrix.

We first compare the qualities of the three approximation schemes: $S_{*}$ defined in (12), $A_{11, k}^{-1}$, and $S^{(2)}$ defined in (16), by using the Berkeley image database. It contains 300 images with ground truth segmentation results. As in [7], we construct a three-layer graph $G^{*}$ with the same setting for mean-shift parameters and $\theta_{g}$. The neighborhood radius $\alpha$ is set to 3 pixels. Following [7], we also manually chose the number of segments for each image. The inter-layer edge weight $\gamma$ in (17) is empirically chosen as 3 . For quantitative evaluation, the following four measures are used (as in [7]): Probabilistic Rand Index (PRI), Variation of Information (VoI), Global Consistency Error (GCE), and Boundary Displacement Error (BDE). For PRI, the larger the better, while for the other three, the smaller the better.

Table 6 shows the results. As can be seen, $S_{*}$ outperforms the other two on all four measures. For benchmark- 


\begin{tabular}{|c|c|c|c|r|r|}
\hline \multirow{2}{*}{ data } & method & \multirow{2}{*}{ accuracy $(\%)$} & \multirow{2}{*}{ NMI } & time $(\mathrm{sec})$ \\
\cline { 5 - 6 } & & & & eig & total \\
\hline \hline \multirow{5}{*}{ RCV1 } & psc (orthogonalized) & $\mathbf{2 5 . 0 5} \pm \mathbf{0 . 6 0}$ & $0.255 \pm 0.007$ & 86.7 & 121.5 \\
& ours (orthogonalized) & $24.72 \pm 0.85$ & $0.253 \pm 0.008$ & $\mathbf{5 . 8}$ & $\mathbf{2 7 . 4}$ \\
& psc (un-orthogonalized) & $22.98 \pm 0.95$ & $0.239 \pm 0.009$ & 30.7 & 64.2 \\
& ours (un-orthogonalized) & $22.54 \pm 1.23$ & $0.236 \pm 0.011$ & $\mathbf{4 . 9}$ & $\mathbf{2 6 . 5}$ \\
\hline \multirow{5}{*}{ ImageNet } & psc (orthogonalized) & $\mathbf{1 0 . 5 6} \pm \mathbf{0 . 0 8}$ & $\mathbf{0 . 1 9 6} \pm \mathbf{0 . 0 0 2}$ & 83.9 & 179.2 \\
& ours (orthogonalized) & $\mathbf{1 0 . 5 6} \pm \mathbf{0 . 1 0}$ & $\mathbf{0 . 1 9 6} \pm \mathbf{0 . 0 0 2}$ & $\mathbf{5 . 8}$ & $\mathbf{8 5 . 8}$ \\
& psc (un-orthogonalized) & $9.42 \pm 0.31$ & $0.169 \pm 0.003$ & 28.2 & 114.0 \\
& ours (un-orthogonalized) & $9.18 \pm 0.11$ & $0.163 \pm 0.002$ & $\mathbf{5 . 4}$ & $\mathbf{8 5 . 3}$ \\
\hline
\end{tabular}

Table 4. Clustering results on the medium-sized data sets.

\begin{tabular}{|c|c|c|c|r|r|}
\hline \multirow{2}{*}{ \#samples used } & \multirow{2}{*}{ method } & \multirow{2}{*}{ accuracy $(\%)$} & \multirow{2}{*}{ NMI } & \multicolumn{2}{|c|}{ time $(\mathrm{sec})$} \\
\cline { 5 - 6 } & & & & eig & total \\
\hline \hline $1,000,000$ & psc & $78.68 \pm 0.18$ & $0.555 \pm 0.002$ & 245.0 & 391.8 \\
$1,000,000$ & ours & $79.07 \pm 0.62$ & $0.564 \pm 0.002$ & $\mathbf{4 . 9}$ & $\mathbf{1 0 1 . 5}$ \\
\hline $4,130,460$ & ours & $77.91 \pm 0.78$ & $0.551 \pm 0.002$ & $\mathbf{1 3 . 4}$ & $\mathbf{4 0 7 . 0}$ \\
\hline
\end{tabular}

Table 5. Clustering results on the MNIST8M data set.

ing, we also show the results reported in [7], which are comparable to ours.

\begin{tabular}{|c|c|c|c|c|}
\hline method & PRI & VoI & GCE & BDE \\
\hline \hline ours $\left(S_{*}\right)$ & $\mathbf{0 . 8 2 0 5}$ & 1.9517 & 0.1998 & $\mathbf{1 2 . 0 9}$ \\
ours $\left(A_{11, k}\right)$ & 0.7814 & 2.4519 & 0.2718 & 13.61 \\
ours $\left(S^{(2)}\right)$ & 0.7598 & 2.6852 & 0.3093 & 14.38 \\
\hline Kim et al. [7] & 0.8146 & $\mathbf{1 . 8 5 4 5}$ & $\mathbf{0 . 1 8 0 9}$ & 12.21 \\
\hline
\end{tabular}

Table 6. Comparison of different $S$ 's and the method in [7] on the Berkeley image database.

To further demonstrate the scalability of the proposed method, we perform segmentation on several very large images downloaded from the web. We manually select the parameter $\alpha$ and $\gamma$ for each image. Results are shown in Figure 2.The first row is the original image and the second row shows the segmentation results and the corresponding computation time. As can be seen, on the $4752 \times 3168 \mathrm{im}-$ age, computing the eigenvectors only takes 23 seconds.

\section{Conclusion}

In this paper, we proposed a time- and space-efficient spectral clustering algorithm. We directly estimate the rank$k$ approximation of $M=I-L$ ( $L$ is the normalized Laplacian) to avoid storing the sampled affinity matrix, which allows the proposed algorithm scaling to very large scale data sets. A general procedure is also proposed to orthogonalize the approximated eigenvectors suitable for both psd and not psd affinity matrices. We performed extensive experiments to show the accuracy and time efficiency of our algorithms and demonstrated the scalability on spectral clustering with several millions data points and image segmentation with 10 million pixels scale.

\section{Acknowledgments}

This research was supported in part by the $\mathrm{Na}$ tional Natural Science Foundation of China (Grant No. 90820018),the National Basic Research Program of China (Grant No. 2009CB320901), the Science and Technology Commission of Shanghai Municipality (Grant No. 09511502400), and the Research Grants Council of the Hong Kong Special Administrative Region (Grant 614508).

\section{References}

[1] F. Bach and M. Jordan. Learning spectral clustering, with application to speech separation. Journal of Machine Learning Research, 7:1963-2001, 2006.

[2] W. Chen, Y. Song, H. Bai, C. Lin, and E. Chang. Parallel spectral clustering in distributed systems. IEEE Transactions on Pattern Analysis and Machine Intelligence, 2010.

[3] D. Comaniciu and P. Meer. Mean shift: A robust approach toward feature space analysis. IEEE Transactions on Pattern Analysis and Machine Intelligence, 24(5):603-619, 2002.

[4] P. Drineas and M. Mahoney. On the Nyström method for approximating a Gram matrix for improved kernel-based learning. Journal of Machine Learning Research, 6:2153-2175, 2005.

[5] F. Fouss, A. Pirotte, J.-M. Renders, and M. Saerens. Random-walk computation of similarities between nodes of a graph with application to collaborative recommendation. IEEE Transactions on Knowledge and Data Engineering, 19:355-369, March 2007.

[6] C. Fowlkes, S. Belongie, F. Chung, and J. Malik. Spectral grouping using the Nyström method. IEEE Transactions on 


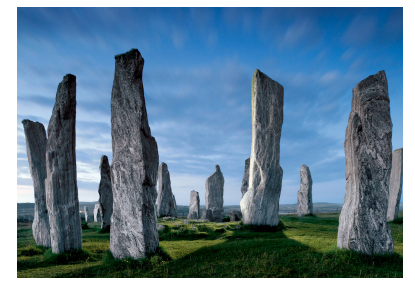

(a) $1.8 \times 10^{6}$ pixels, $1600 \times 1122$

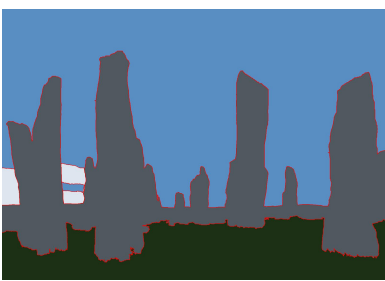

(e) 4 segments, $5.9 \mathrm{sec}$.

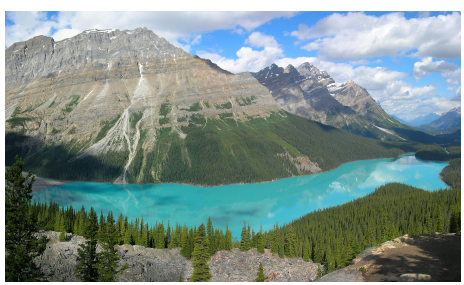

(b) $10^{7}$ pixels, $4152 \times 2467$

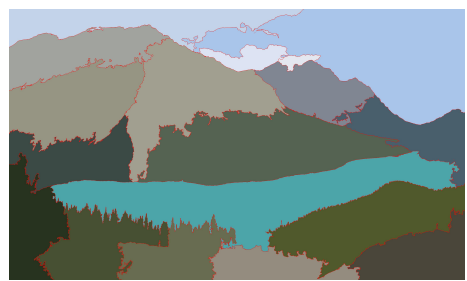

(f) 18 segments, $18.9 \mathrm{sec}$.

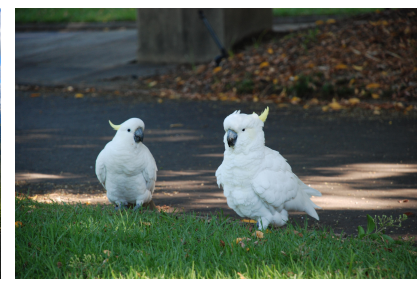

(c) $10^{7}$ pixels, $3872 \times 2592$

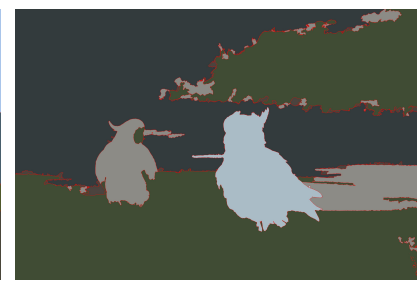

(g) 5 segments, $16.8 \mathrm{sec}$.

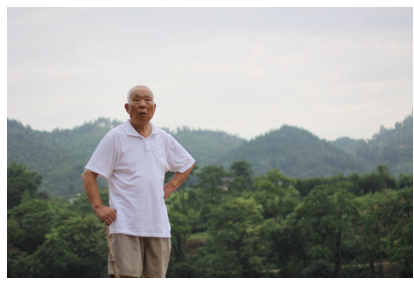

(d) $1.5 \times 10^{7}$ pixels, $4752 \times 3168$

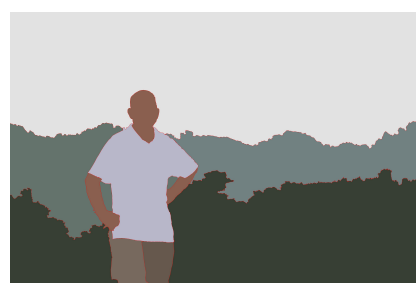

(h) 8 segments, $22.6 \mathrm{sec}$.

Figure 2. Image segmentation on very large images. The first row shows the image, its size, and the number of pixels. The second row shows the segmentation result and the time used on computing the eigenvectors.

Pattern Analysis and Machine Intelligence, pages 214-225, 2004.

[7] T. Kim and K. Lee. Learning full pairwise affinities for spectral segmentation. In CVPR, pages 2101-2108, 2010.

[8] S. Kumar, M. Mohri, and A. Talwalkar. On sampling-based approximate spectral decomposition. In $I C M L$, pages 585$592,2009$.

[9] D. Lewis, Y. Yang, T. Rose, and F. Li. Rcv1: A new benchmark collection for text categorization research. Journal of Machine Learning Research, 5:361-397, 2004.

[10] B. Long, Z. M. Zhang, X. Wú, and P. S. Yu. Spectral clustering for multi-type relational data. In ICML, pages 585-592, 2006.

[11] A. Ng, M. Jordan, and Y. Weiss. On spectral clustering: Analysis and an algorithm. In NIPS, pages 849-856, 2001.

[12] J. Shi and J. Malik. Normalized cuts and image segmentation. IEEE Transactions on Pattern Analysis and Machine Intelligence, 22(8):888-905, 2002.

[13] A. Strehl and J. Ghosh. Cluster ensembles - A knowledge reuse framework for combining multiple partitions. Journal of Machine Learning Research, 3:583-617, 2003.

[14] M. Swain and D. Ballard. Color indexing. International Journal of Computer Vision, 7(1):11-32, 1991.

[15] A. Talwalkar, S. Kumar, and H. Rowley. Large-scale manifold learning. In $C V P R$, pages 1-8, 2008.

[16] U. Von Luxburg. A tutorial on spectral clustering. Statistics and Computing, 17(4):395-416, 2007.

[17] C. Williams and M. Seeger. Using the Nyström method to speed up kernel machines. In NIPS, 2001.

[18] D. Yan, L. Huang, and M. Jordan. Fast approximate spectral clustering. In KDD, pages 907-916, 2009.

\section{A. Proof of Proposition 1}

First we introduce the following lemma.
Lemma 3. Assume that graph $G$ has no more than $k$ connected components, then $\operatorname{diag}\left(D^{\frac{1}{2}} M_{k} D^{\frac{1}{2}} 1\right)=D$.

Proof. Let $L=I-M$ be the normalized Laplacian matrix of $W$. Then $L$ is psd and has exactly $c$ zero eigenvalues, where $c$ is the number of connected components of $G$ [16]. Therefore, the $c$ largest eigenvalues of $M$ are equal to 1 . As $M_{k}$ keeps the $k$ largest eigenvalues of $M$ and $k \geq c, M_{k}$ also keeps the eigenspace which is spanned by the eigenvectors with eigenvalue 1 . As $M\left(D^{\frac{1}{2}} 1\right)=D^{-\frac{1}{2}} W 1=D^{\frac{1}{2}} 1$, so $D^{\frac{1}{2}} 1$ is an eigenvector of $M$ with eigenvalue 1 . Thus, it is also an eigenvector of $M_{k}$. Hence, $M_{k}\left(D^{\frac{1}{2}} 1\right)=D^{\frac{1}{2}} 1$ and the result follows.

Proof. (Proposition 1) Let $D_{*}=\operatorname{diag}\left(A_{11} 1\right)$ and $M_{*}=$ $D_{*}^{-\frac{1}{2}} A_{11} D_{*}^{-\frac{1}{2}}$. Then

$$
\begin{aligned}
& D_{*}^{-\frac{1}{2}} M_{*, k}^{-1} D_{*}^{-\frac{1}{2}} A_{11} 1 \\
& =A_{11}^{-1} D_{*}^{\frac{1}{2}}\left(D_{*}^{-\frac{1}{2}} A_{11} D_{*}^{-\frac{1}{2}}\right) M_{*, k}^{-1}\left(D_{*}^{-\frac{1}{2}} A_{11} D_{*}^{-\frac{1}{2}}\right) D_{*}^{\frac{1}{2}} 1 \\
& =A_{11}^{-1} D_{*}^{\frac{1}{2}} M_{*, k} M_{*, k}^{-1} M_{*, k} D_{*}^{\frac{1}{2}} 1 \\
& =A_{11}^{-1} D_{*}^{\frac{1}{2}} M_{*, k} D_{*}^{\frac{1}{2}} 1=A_{11}^{-1} A_{11} 1=1,
\end{aligned}
$$

where the second last equation is from Lemma 3. Therefore, $\hat{A}_{: 1} 1=A_{: 1} D_{*}^{-\frac{1}{2}} M_{*, k}^{-1} D_{*}^{-\frac{1}{2}} A_{11} 1=A_{: 1} 1$.

\section{B. Proof of Proposition 2}

Proof. Note that $U \Lambda U^{T}=$ $\left(U V \Sigma^{-\frac{1}{2}}\right) \tilde{V} \tilde{\Lambda} \tilde{V}^{T}\left(U V \Sigma^{-\frac{1}{2}}\right)^{T}=\tilde{U} \tilde{\Lambda} \tilde{U}^{T}$. Moreover, $\tilde{U}$ is orthogonal as $\tilde{U}^{T} \tilde{U}=\tilde{V}^{T} \Sigma^{-\frac{1}{2}} V^{T}\left(V \Sigma V^{T}\right) V \Sigma^{-\frac{1}{2}} \tilde{V}=$ $\tilde{V}^{T} \tilde{V}=I$. 